
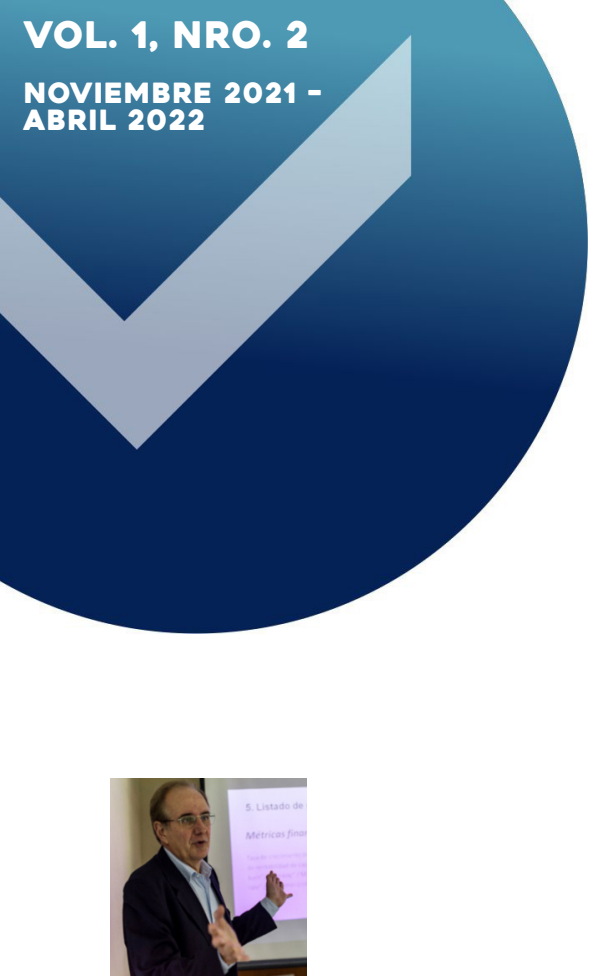

Ricardo Villarmarzo

Ex Profesor Titular de Auditoría y de Informes de Contador Público. Facultad de Ciencias Económicas y de Administración. Universidad de la República. Uruguay.

$\bowtie$ ricardo.villarmarzo@gmail.com

\title{
ENSAYO
}

\section{ANÁLISIS DE LAS CUESTIONES CLAVES DE LA AUDITORÍA Y SUS IMPLICANCIAS PARA LA PRÁCTICA PROFESIONAL}

\author{
ANALYSIS OF KEY MATTERS IN AUDITING AND \\ THEIR IMPLICATIONS FOR THE PROFESSIONAL \\ PRACTICE
}

\section{RESUMEN}

Los informes profesionales de auditoría emitidos en determinado tipo de circunstancias tienen que contemplar la inclusión de las denominadas "cuestiones claves de la auditoría". El reciente comienzo de la aplicación de la Norma Internacional de Auditoría (NIA) 701 en la práctica en algunos países de la región hace oportuno analizar en forma esquemática ciertos aspectos relevantes a ser contemplados en el ejercicio profesional. A esos efectos, el artículo procura resumir los elementos sustanciales de esa normativa, algunos de los aspectos más importantes a evaluar en su aplicación, así como ejemplos de redacción extraídos de la realidad en Uruguay como referencia inicial. Su aplicación tiene potencial efecto en todas las etapas del examen profesional realizado por el auditor: planificación del trabajo; integración del equipo; evaluación de riesgos; aplicación de procedimientos en cuanto a alcance, naturaleza y oportunidad; derivación de las conclusiones; redacción del informe; relacionamiento con la dirección de la empresa y responsabilidades frente a los usuarios.

\section{ABSTRACT}

Professional auditors' reports issued under certain circumstances must consider the inclusion of what is known as "key audit matters".

The recent start of the application in practice of the corresponding international standard of auditing in some of our countries determines that it is adequate to briefly analyse certain relevant aspects to be considered in the professional practice.

In this respect, the article tries to summarise the most important elements of this standard, its relevant characteristics, some of the significant aspects to be evaluated during its application, as well as some wording examples used in the Uruguayan reality as initial reference.

\section{PALABRAS CLAVE}

auditoría - cuestiones claves - experiencias - reflexiones.

\section{KEYWORDS}

audit - key matters - experiences - reflections.

ANÁLISIS DE LAS CUESTIONES CLAVES DE LA AUDITORÍA Y SUS IMPLICANCIAS PARA LA PRÁCTICA PROFESIONAL

\section{AUTOR:}

Ricardo Villarmarzo
RECIBIDO:

2 de septiembre, 2021

APROBADO:

24 de septiembre, 2021

\section{AUDITAR}

PRIMERA REVISTA ARGENTINA

EXCLUSIVA SOBRE AUDITORÍA
DOI: https://doi.org/10.24215/27188647e009 CÓDIGO JEL: M42

ISSN: 2718-8647

http://revistas.unlp.edu.ar/auditar

\section{ENTIDAD EDITORA:}

Instituto de Investigaciones y Estudios

Contables, Facultad de Ciencias Económicas, Universidad Nacional de La Plata 


\section{¿DE QUÉ SE TRATA ESTA NOVEDAD NOR- MATIVA?}

Los informes profesionales de auditoría sobre información económico-financiera expresada en estados contables han tenido importantes cambios en los últimos años, a partir de la aprobación de diversas normas internacionales por la Federación Internacional de Contadores (IFAC - por su sigla en idioma inglés). En muchos países alrededor del mundo se ha avanzado en la aplicación de la nueva normativa, que contempla cambios en la estructura del informe, en su ordenamiento, en la profundidad y en la extensión de aspectos vinculados con la tarea realizada y con las responsabilidades de los emisores y de los auditores y diversas temáticas asociadas. Un aspecto muy relevante ha sido la introducción -en forma obligatoria, para el caso de informes emitidos en relación con compañías que cotizan en los mercados de valores y otros casos específicos- de las denominadas "cuestiones claves de la auditoría" (CCA).

La razón fundamental que ha llevado a estas modificaciones ha sido el intento por reducir la "brecha de expectativas" entre los emisores de la información, los usuarios de los informes y los profesionales a cargo de llevar a cabo los exámenes respectivos.

El presente artículo procura contemplar en su desarrollo los siguientes aspectos sustanciales:

a) ¿Cuáles son los elementos sustanciales de la normativa internacional aplicables?

b) ¿De qué manera están comenzando a ser contemplados en la realidad del Uruguay?

c) ¿Cuáles son los problemas más relevantes que surgen en el proceso de aplicación de este nuevo concepto en el ejercicio profesional de auditoría?

d) ¿De qué manera se pueden encaminar soluciones para contemplar dichas dificultades?

e) ¿Cómo debe ser la redacción sobre las CCA en el dictamen profesional?

f) ¿Qué elementos de juicio habrá que tomar en cuenta en los primeros tramos de la experiencia de su implementación?

\section{LA NORMATIVA DE LA FEDERACIÓN INTER- NACIONAL DE CONTADORES}

La norma de auditoría que contempla la problemática a nivel de IFAC es la Norma Internacional del Auditoría (NIA) 701

- "Comunicación de cuestiones claves de la auditoría en el informe de auditoría emitido por un auditor independiente" (IFAC, 2015). Su objetivo es dar pautas de referencia respecto a qué debe comunicar el auditor en su informe, así como respecto a su forma y su contenido. Se intenta otorgar más transparencia sobre el examen profesional realizado, de forma que los usuarios de los estados financieros estén en mejores condiciones de comprender cuáles fueron los temas más relevantes en el trabajo profesional llevado a cabo.

De ninguna manera debe utilizarse su comunicación como una alternativa a las explicaciones que deben incluirse en los estados financieros y en sus notas, ni para evitar emitir una opinión modificada sobre ellos, ni como manera de informar problemas de "empresa en marcha", ni como una opinión sobre cuestiones específicas de la información incluida. Emitiéndose opiniones calificadas 0 adversas, igualmente corresponde incluir CCA. Se aclara que no debe hacerse referencia a ellas cuando el profesional se abstiene de opinar sobre los estados financieros examinados. También se destaca que la inclusión de CCA no impide la existencia de párrafos de énfasis en el informe profesional.

\section{Definición}

La norma define las cuestiones claves de la auditoría como aquellas que, según el juicio profesional del auditor, fueron de mayor significación en la auditoría de los estados financieros del ejercicio examinado y se seleccionan entre las que fueron comunicadas a los responsables de la empresa.

\section{Determinación}

Para definir las cuestiones que deben ser incluidas en el informe el auditor, se deben tomar en cuenta los siguientes aspectos: 
a) las áreas evaluadas como de mayor riesgo de afirmaciones erróneas materiales 0 con riesgos significativos según las pautas de la NIA 315;

b) Ios juicios importantes del auditor relacionados con áreas de los estados financieros que involucran subjetividad de la dirección, incluyendo en particular las estimaciones contables identificadas como de alta incertidumbre;

c) el efecto sobre el examen de auditoría de hechos o transacciones significativos ocurridos durante el período examinado.

\section{Comunicación}

Cada una de las cuestiones debe ser incluida en el dictamen profesional bajo un subtítulo apropiado, en una sección separada del informe titulada "Cuestiones claves de la auditoría".

La descripción introductoria de esa sección debe aclarar el concepto de esta temática y mencionar que fueron consideradas en el contexto de los estados financieros en su conjunto a los efectos de la expresión de la opinión profesional. A su vez, debe explicitar que no constituyen una opinión aislada sobre ellos. Adicionalmente, situaciones que requieren la modificación de la opinión profesional no pueden ser incluidas entre las CCA.

La descripción de cada asunto debe incluir una referencia a la correspondiente explicación en los estados financieros, si correspondiese, y establecer:

a) por qué el asunto fue considerado como uno de los más significativos en la auditoría;

b) cómo fue encarado el problema en cuestión durante la realización del examen profesional.

Cabe señalar que, si no existieran CCA a ser incluidas en el informe del auditor, el párrafo respectivo existirá indicando esa situación.

Nunca se puede dejar de lado un tema a incluir por el hecho de que las notas a los estados contables de la empresa contengan explicaciones relacionadas con la situación en cuestión ni viceversa.

\section{LA REALIDAD EN URUGUAY}

A nivel local, se aprobó la aplicación de la nueva norma internacional por parte del Colegio de Contadores, Economistas y Administradores del Uruguay, y el Banco Central del Uruguay definió que para las empresas "abiertas" bajo su control regiría a partir de ejercicios cerrados a partir del 15 de diciembre de 2019.

Han comenzado a difundirse informes de auditoría en los cuales se han incluido párrafos referidos a CCA en compañías alcanzadas por la nueva normativa, siendo los casos más notorios las instituciones bancarias y las entidades que cotizan en la Bolsa de Valores de Montevideo.

En el caso de instituciones bancarias, por ejemplo, los informes de auditoría relacionados con los estados contables al 31 de diciembre de 2020 han incorporado CCA vinculadas con el deterioro de los créditos y los sistemas de tecnología de la información y su actualización, entre otros (ver ANEXOS).

\section{PROBLEMAS PRÁCTICOS PARA EL EJERCICIO PROFESIONAL}

¿Qué factores influyen en la identificación de las "cuestiones claves de la auditoría"?

La normativa profesional define las CCA y da algunas pautas al respecto, pero siempre será parte del juicio profesional subjetivo la forma de su aplicación en cada examen llevado a cabo. Es muy probable que profesionales diferentes pudieran tener visiones distintas en cuanto al mismo problema, más allá de que las "cuestiones claves de la auditoría" deben ser específicas de cada empresa. Por ello, se deben agotar las instancias para eliminar en todo lo posible los impactos en la evaluación que no respondan a evidencia estrictamente objetiva.

Puede ocurrir que en una industria o en un área de actividad haya situaciones que sean comunes de encontrar en muchas 
de las organizaciones que actúan en ese sector, más allá de la especificidad vinculada a cada empresa concreta.

Probablemente la identificación de las CCA está asociada a responderse profesionalmente preguntas tales como qué aspectos de la operativa pueden ir mal en una empresa, cuyas consecuencias tengan necesidad de ser expuestas y qué aspectos de la información contable tienen mayor grado de subjetividad en su expresión conceptual y numérica. Ello incidirá en el tipo y en la cantidad de trabajo a realizar en el examen profesional.

En principio, pueden asociarse a temáticas que tienen relación con hechos pasados, pero también existe la posibilidad de que se vinculen a acontecimientos del futuro.

$\mathrm{Y}$ un aspecto complementario para nada menor refiere a cómo influyen en este proceso de identificación la "materialidad" y la generalidad en los estados contables. No cabe duda de lo primero: se tiene que estar hablando de algo significativo en el contexto de la información presentada. Respecto al segundo elemento, seguramente es trascendente que el asunto pudiera impactar el patrimonio, el resultado, el capital de trabajo y otras variables importantes similares.

\section{Potenciales situaciones a ser reveladas como "cuestiones claves de la auditoría"}

Potencialmente, las situaciones que se pueden dar en la práctica del ejercicio profesional no son fáciles de clasificar y menos de acotar. En primer término, es claro que las CCA estarán generalmente vinculadas con las áreas de riesgo identificadas y definidas en la etapa de planificación del examen profesional.

La dosis de subjetividad asociada a los rubros de los estados contables y a la forma de determinación y valuación de los saldos respectivos seguramente es un aspecto de incidencia trascendente (por ejemplo, todas las partidas que implican estimaciones 0 afines). Asimismo, se entiende que proble- mas relacionados con deficiencias de control interno con potencial impacto relevante también podrían constituirse en cuestiones de esta índole. En particular, se consideran potenciales temáticas a considerar en esta evaluación las transacciones con partes relacionadas u otras de importancia que se encuentren fuera del giro habitual de los negocios o sean inusuales.

De la misma manera, la forma en la cual la tecnología de la información y ciertas problemáticas digitales pueden tener impacto para el desarrollo de la gestión empresarial también haría de sus eventuales consecuencias motivo para la inclusión como "cuestiones claves de la auditoría". En particular, la norma menciona como un posible ejemplo la implementación de un nuevo sistema de tecnología informática en la organización.

Se discute si serán objeto de inclusión problemáticas con repercusión empresarial relacionadas con la sostenibilidad o el cambio climático y afines.

No corresponde mencionar en el informe profesional actualizaciones sobre CCA incluidas en ejercicios previos, salvo que mantengan todavía vigencia.

Es importante destacar que la normativa plantea expresamente que no es parte de la idea la existencia de largas listas de CCA incluidas en el informe profesional, ya que atentan contra la noción de significatividad que se pretende lograr. En la aplicación del juicio profesional, debe tomarse en consideración para su selección el tamaño y la complejidad de la empresa, la naturaleza de los negocios y del contexto en que se opera y los hechos y circunstancias del compromiso de auditoría concreto.

\section{Aspectos particulares a considerar para la definición de la} inclusión de "cuestiones claves de la auditoría"

Por supuesto, un paso a contemplar es la comunicación de las situaciones a la Dirección y la Gerencia del cliente, valorando adecuadamente sus opiniones y la evidencia que se aporte para sostenerlas. 
Cabe señalar que la normativa plantea la posibilidad o la necesidad de relacionar las CCA con explicaciones incluidas por la organización en las notas a los estados contables, y ese aspecto también es muy relevante en el proceso en cuestión. $\mathrm{Y}$, en tal sentido, la normativa también prevé situaciones a ser contempladas respecto a elementos que pueden ser de carácter confidencial para la marcha de la empresa y que no deberían desglosarse en la descripción de las CCA por parte del profesional.

\section{Consecuencias para los profesionales en su ejercicio}

Es altamente probable que a futuro exista un mayor escrutinio de las partes interesadas respecto al trabajo llevado a cabo por el auditor debido a la inclusión de esas "cuestiones claves de la auditoría". Inicialmente, también se percibe un riesgo mayor en cuanto a la responsabilidad del profesional y la posibilidad de ser demandado legalmente 0 de verse involucrado en problemas de similares características. En algunos ámbitos profesionales se considera que el auditor requerirá dedicar más tiempo y aplicar más procedimientos de los habituales anteriormente y, en función de ello, se agregaría un elemento adicional a "las tensiones" sobre los honorarios correspondientes.

\section{POTENCIALES ENFOQUES PARA CONTEMPLAR EN ESTAS SITUACIONES}

El auditor deberá llevar adelante su examen con más precauciones y cuidado profesional del que ya tuvo en el pasado.

Entre las instancias del trabajo a reforzar especialmente aunque forman parte del enfoque habitual del examen profesional-, se encuentran, entre otras:

- Integración del equipo profesional asignado a las tareas e involucramiento de personas con la experiencia apropiada en la industria, contemplando la participación de especialistas, cuando corresponda, para el análisis complementario 0 independiente del trabajo realizado.
- Identificación detallada y minuciosa de los riesgos del negocio y de su potencial impacto en la auditoría.

- Planificación detallada de todas las etapas de ejecución del examen.

- Evaluación sostenida de la evidencia obtenida y de la contundencia de las conclusiones alcanzadas.

- Especial cuidado en la documentación de los procedimientos aplicados y de la evidencia obtenida.

- Intercambio fluido y objetivo con la Dirección y la Gerencia de los elementos determinantes de las CCA.

- Evaluación objetiva final.

- Redacción cuidadosa de las situaciones en los párrafos correspondientes del informe del auditor, contemplando todos los aspectos específicos establecidos en la normativa.

La atención del profesional a cargo del examen debe centrarse especialmente en las áreas de riesgo de potenciales afirmaciones erróneas materiales en los estados financieros y en el diseño y en la ejecución de procedimientos de auditoría que respondan a ellos, obteniendo evidencia suficiente y adecuada para respaldar la opinión a emitir.

Existe una relación directa entre la importancia de los riesgos y la cantidad de trabajo a realizar y de evidencia a disponer. En particular, la evidencia deberá ser más numerosa y relevante o creíble, implicando por ejemplo la obtención de aquella que depende de terceros 0 validándola en base a fuentes independientes.

\section{LA REDACCIÓN EN EL INFORME}

\section{Consideraciones generales}

Los aspectos formales y el contenido de las CCA están establecidos en los estándares aprobados. Allí se brindan pautas referidas a la descripción de los temas y a la redacción respectiva.

Las preguntas mínimas que deberían quedar respondidas con la inclusión de los temas serían: a) ¿qué?, b) ¿por qué?, 
c) ¿cómo? y d) ¿dónde? 0 sea, qué constituye el problema en cuestión; por qué debe ser considerado tal, destacando los procedimientos aplicados para llegar a esa conclusión; cómo afectan a la gestión de la empresa; dónde impactan en los estados contables.

Una consideración muy importante a tener en cuenta refiere a que la redacción debe ser realizada en lenguaje no técnico, para que las "cuestiones claves de la auditoría" puedan ser comprendidas en toda su dimensión y sin dificultades por los usuarios habituales de los informes profesionales.

Seguramente esta novedad determinará que los informes profesionales de auditoría sean cada vez más extensos y demanden una lectura muy atenta por parte de las distintas partes interesadas.

\section{Consideraciones formales}

La sección de CCA debe ser incluida en forma inmediata a continuación de la opinión del auditor y de sus fundamentos para darle la debida relevancia.

El orden de presentación de los temas también queda a decisión del juicio profesional del auditor actuante. La norma menciona entre las posibilidades la inclusión según su importancia relativa 0 en función de la manera en la cual aparecen los rubros en los estados financieros y sus notas. Si es necesario, se encabezará cada asunto con el correspondiente subtítulo.

Asimismo, en caso de estados contables comparativos, la redacción debe dejar en claro que los CCA refieren únicamente a las correspondientes al ejercicio actual.

La descripción no debe ser una reiteración de lo que ya puede haber sido incluido en las notas a los estados contables, pero una referencia a ellas puede ser útil para una mejor comprensión de la problemática en cuestión (por ejemplo, estimaciones contables, "empresa en marcha").
La redacción debe ser lo más específica posible para el caso concreto de la empresa, más allá de que el tipo de problema pueda ser relativamente común en la industria o en el área de negocios que se lleva a cabo. Se recomienda evitar redacciones estandarizadas de CCA que sean de frecuente ocurrencia en circunstancias determinadas.

Se debe incluir un resumen de la forma en la cual el auditor encaró el análisis y la consideración práctica del asunto en su examen profesional, refiriéndose a los procedimientos aplicados, sus resultados y las observaciones relevantes que hayan surgido. Se necesita ser claro en cuanto a cómo se solucionó el tema y nunca redactar de forma que pueda malinterpretarse que se emite una opinión parcial al respecto o confusiones similares.

\section{CONCLUSIONES}

Aunque la experiencia determinará con el transcurso del tiempo la real utilidad práctica del impacto de esta normativa, en primera instancia, su aplicación en los informes de auditor independiente de las organizaciones en las cuales corresponda contemplarla debería reforzar la utilidad para los usuarios de las conclusiones alcanzadas y la claridad del alcance del examen realizado.

Por su parte, dará un respaldo adicional al auditor en la aplicación de su juicio profesional ante cuestiones que merezcan ser destacadas dentro del contexto de la normativa y en eventuales instancias de revisiones de calidad independientes 0 por parte de organismos de contralor.

Con el transcurso del tiempo probablemente se extenderá su aplicación de manera más generalizada, a la vez que quizás surjan aclaraciones complementarias o guías de aplicación más detalladas basadas en la experiencia recogida. 


\section{REFERENCIAS}

Banco de la República Oriental del Uruguay. (2021). Estados financieros correspondientes al 31 de diciembre de 2020 e informe de auditoría independiente. https://www.brou. com.uy/documents/20182/52654/auditorias-2020.pdf/ f52eb27d-abec-4c47-8892-0875d9a051fe

International Federation of Accountants. (2015). International standard on auditing 701 - Communicating key audit matters in the independent auditor's report. https:// www.iaasb.org/publications/international-standard-auditing-isa-701-new-communicating-key-audit-matters-independent-auditors-4

Scotiabank Uruguay S.A. (2021). Estados Financieros al 31 de diciembre de 2020. https://cdn.agilitycms.com/scotiabank-uruguay/pdf/acerca-de/estados-contables/ScotibankUruguayEECCdic2020.pdf

\section{ANEXOS}

Se acompañan a continuación ejemplos de redacción de "cuestiones claves de la auditoría", extraídos de informe emitidos en la realidad uruguaya, para instituciones del sistema bancario.

\section{Ejemplo 1}

Scotiabank - informe del auditor independiente - ejercicio terminado al 31 de diciembre de 2020 - Transcripción (Scotiabank Uruguay S.A., 2021)

\section{Cuestiones Clave de la Auditoría}

Las cuestiones clave de la auditoría son aquellas cuestiones que, según nuestro juicio profesional, han sido de la mayor significatividad en nuestra auditoría de los estados financieros del período actual. Estas cuestiones han sido tratadas en el contexto de nuestra auditoría de los estados financieros en su conjunto y en la formación de nuestra opinión sobre estos, y no expresamos una opinión por separado sobre esas cuestiones.

\section{Deterioro de créditos por intermediación financiera a costo amortizado}

\section{Cuestión clave de auditoría}

El cálculo del deterioro de créditos por intermediación financiera requiere juicio considerable al tratarse de una estimación significativa. Para estimar el deterioro de activos financieros a costo amortizado, el Banco aplica lo establecido en el Anexo 2 de la Comunicación 2020/067 del Banco Central del Uruguay. A efectos de la estimación de su deterioro, como se menciona en la Nota 2.1.5, los créditos por intermediación financiera son clasificados en distintas categorías de riesgo siguiendo criterios objetivos y subjetivos definidos en el Anexo 1 de la mencionada Comunicación y las disposiciones transitorias mencionadas en la Nota 3. Como se menciona en la Nota 3 a los estados financieros, en el mes marzo de 2020 se ha propagado en Uruguay y en el mundo un virus denominado Covid-19, que ha sido declarado como pandemia por la Organización Mundial de la Salud con fecha 11 de marzo de 2020. No es posible estimar el impacto final del mismo, la propagación y extensión en el tiempo de este evento podría afectar la capacidad de pago de los deudores del Banco con el consecuente impacto en las provisiones para riesgos crediticios. La consideración de esta cuestión como clave en nuestra auditoría se basa tanto en la significatividad de la cartera de créditos por intermediación financiera a costo amortizado como en la relevancia y complejidad del cálculo de dicho deterioro, acentuado por la coyuntura actual producto de la pandemia.

\section{Cómo se trató la cuestión en nuestra auditoría}

Nuestro enfoque de auditoría ha incluido tanto la evaluación de los controles relevantes vinculados a los procesos de estimación del deterioro por riesgo de crédito de la cartera de créditos por intermediación financiera a costo amortizado, cómo la realización de pruebas analíticas y de detalle sobre dicha estimación. Nuestros procedimientos relativos al entorno de control se han centrado en las siguientes áreas claves:

- Gobernanza: identificación del marco de gestión de riesgos crediticios y controles relevantes.

Políticas contables: evaluación de su alineación con la regulación contable aplicable.

- Categorización en función de su riesgo de crédito conforme con los criterios establecidos por el Banco Central del Uruguay. Nuestras pruebas de detalle sobre la estimación de provisión por deterioro, tomando en consideración la naturaleza y el alcance de las pruebas de auditoría requeridas por el Banco Central del Uruguay, han sido básicamente las siguientes:

- Para una muestra de clientes, evaluamos su adecuada clasificación y la suficiencia de la provisión específica constituida por el Banco de acuerdo con los criterios establecidos por el Banco Central del Uruguay, incluidas las disposiciones transitorias relativas a Covid-19.

- Para una muestra de garantías computables evaluamos la existencia y valuación de las mismas de acuerdo a lo establecido por el Banco Central del Uruguay.

- Con relación al fondo de previsiones estadísticas, evaluamos su correcta determinación de acuerdo a lo establecido por el Banco Central del Uruguay.

- Finalmente, hemos analizado si la información detallada en las notas a los estados financieros resulta adecuada, de conformidad con los criterios establecidos en el marco de la información financiera aplicable al Banco. 


\section{Ejemplo 2}

Banco de la República Oriental del Uruguay (BROU) - informe del auditor independiente - ejercicio terminado al 31 de diciembre de 2020 Asuntos clave de auditoría

Los asuntos clave de auditoría son aquellos asuntos que, de acuerdo a nuestro juicio profesional, fueron de mayor importancia en nuestra auditoría de los estados financieros consolidados del período actual. Estas cuestiones fueron abordadas en el contexto de nuestra auditoría como un todo, y en la formación de nuestra opinión al respecto, por lo que no representan una opinión por separado sobre estos asuntos. (Banco de la República Oriental del Uruguay, 2020, p. 170)

Deterioro de los riesgos crediticios con el sector no financiero a costo amortizado

Tal como se describe en la Nota 34, los riesgos crediticios por intermediación financiera a costo amortizado con el sector no financiero ascienden a miles de $\$ 227.240 .106$, y la provisión por deterioro asciende a miles de $\$ 17.203 .511$.

De acuerdo a lo detallado en la Nota 2.1.6, el Grupo constituye provisiones por deterioro de acuerdo las normas contables establecidas por el Banco Central del Uruguay, que implican determinar la categoría de riesgo de cada deudor de acuerdo al Anexo 1 de la Comunicación 2020/241, considerar las garantías computables de acuerdo a los Anexo 3 y 4 de dicha Comunicación y los porcentajes de previsiones aplicables según lo que surge del Anexo 2 de la misma.

Tal como surge de la Nota 34.1, se aplican diferentes criterios a la hora de determinar la categoría de riesgo crediticio correspondiente. En general, se aplican los criterios objetivos y subjetivos establecidos por la Normativa del Banco Central del Uruguay, y en algunos casos, dependiendo del monto de endeudamiento, se aplican criterios de evaluación simplificados.

En particular, y tal como se describe en la Nota 3, durante el año 2020, se ha propagado en nuestra región el virus Coronavirus COVID-19 que ha sido calificado como una pandemia por la Organización Mundial de la Salud el 11 de marzo de 2020. A partir de dicho contexto, el Banco Central del Uruguay adoptó un conjunto de medidas que le han permitido a las Instituciones Financieras ciertas flexibilizaciones asociadas con la cartera de créditos.
Dada la importancia cuantitativa de la provisión por deterioro, la actual coyuntura económica, la complejidad que involucra su determinación por la existencia de criterios subjetivos y que se trata de una cifra muy relevante para la evaluación del desempeño financiero y de la gestión del riesgo de crédito, consideramos que se trata de un asunto clave de auditoría.

Nuestros procedimientos efectuados fueron los siguientes:

- Evaluación de las políticas y procedimientos de evaluación de riesgo crediticio aplicadas por el Grupo, analizando que las mismas se encuentran alineadas con los requerimientos del Banco Central del Uruguay.

- Evaluación del proceso de determinación de calificaciones subjetivas y la existencia de criterios de aprobación por parte de los diferentes niveles de autoridad del Grupo, en aquellos casos aplicables, considerando el endeudamiento de los clientes.

- Evaluación del diseño y la implementación de los controles internos asociados a este proceso (determinación objetiva de la categoría de riesgo en función del atraso en las operaciones, el cálculo automático de provisiones, la determinación de la categoría subjetiva de los deudores), tanto manuales como automáticos, y en algunos casos que consideramos necesario, hemos probado la efectividad operativa de los mismos.

- Siguiendo con los lineamientos establecidos por el Banco Central del Uruguay, hemos determinado una muestra selectiva de clientes, para los cuales hemos realizado procedimientos de auditoría tendientes a analizar que la categoría de riesgo asignada, el riesgo crediticio, las garantías computables y la provisión por deterioro determinado por el Grupo, fueran adecuadas de acuerdo a la normativa del Banco Central del Uruguay aplicable.

- Evaluación de la razonabilidad de las revelaciones relacionadas con este asunto en los estados financieros, de acuerdo a lo establecido por el marco contable definido por el Banco Central del Uruguay.

- Evaluación de la razonabilidad de la revelación realizada por el Grupo respecto del impacto de la pandemia generada por el COVID-19 en la determinación de la previsión por deterioro, incluida en la Nota 3.

(Banco de la República Oriental del Uruguay, 2021, p. 172). 